\title{
Coupling the Monte-Carlo Method with Semi-Analytical Solutions of the Boltzmann Transport Equation
}

\author{
S. C. Brugger ${ }^{1}$, V. Peikert ${ }^{2}$, and A. Schenk ${ }^{2,3}$ \\ ${ }^{1}$ Numerical Solutions GmbH, Regensdorferstr. 155, CH-8049 Zürich, Switzerland \\ ${ }^{1}$ e-mail: brugger@numericalsolutions.ch,Tel/Fax: +41 445867684 \\ ${ }^{2}$ Integrated Systems Laboratory, Swiss Fed. Inst. of Technology, Gloriastr. 35, CH-8092 Zürich, Switzerland \\ 3 e-mail: schenk@iis.ee.ethz.ch, Tel: +41 44632 6689, Fax +41 446321194
}

\begin{abstract}
This paper presents an important improvement of the current-based one-particle Monte-Carlo method. By regionwise coupling a semi-analytical solution of the Boltzmann equation to a full solution, the usage of computationally intensive Monte Carlo Boltzmann solvers can be limited to only those regions where they are needed. The advantages and drawbacks of this new method are reviewed and the problems arising from the coupling scheme are discussed.
\end{abstract}

\section{INTRODUCTION}

The Monte Carlo (MC) method is a well known approach to solve the Boltzmann transport equation (BTE). It allows to compute results with usable accuracy, without requiring as much memory as direct methods. However, at low and medium electric fields and in moderately to highly doped regions the MC method is rather innefficient due to the very short mean free paths of the simulated particles. In [1] a semianalytical method has been described which allows to solve the BTE in all orders in the driving forces (DF). This semianalytical method can be coupled with the MC technique and can advantageously replace the MC method in the problematic regions.

After a concise theoretical introduction, the coupling between the semi-analytical method and the MC method is carefully described. Then, a simple example is chosen to illustrate the advantages and drawbacks of the new coupling scheme. It is then shown how this hybrid method allows to understand which DF are mainly responsible for the behavior of the transport parameters inside a device. Problems arising from the discretization of the first Brillouin zone and from the stochastic nature of the MC method are then discussed. A short summary will conclude the discussion.

\section{THEORY}

\section{A. Semi-analytical solution}

In the following we consider only Boltzmann statistics because Monte Carlo is a very inefficient method when solving for Fermi statistics. However, a semi-analytical solution can also be derived for Fermi statistics.
Multiplying the space-inhomogeneous BTE with the inverse scattering operator (ISO) [2], using the important property (B.18, p. 250 in [3]) and rearranging the terms leads to

$$
h=-\beta \nabla_{r} \psi \cdot S_{\vec{v}}^{-1}-\beta \nabla_{r} \psi \cdot S_{\vec{v} h}^{-1}+\frac{q}{\hbar} \nabla_{r} \phi \cdot S_{\nabla_{k} h}^{-1}+S_{\vec{v} \cdot \nabla_{r} h}^{-1},
$$

where the distribution function $f$ has been rewritten as $f=$ $f_{e q}(1+h), \beta:=\frac{q}{k_{B} T}, \psi$ is the quasi-Fermi potential, and $\phi$ is the electrostatic potential. The quasi-Fermi potential is defined as $\psi:=-\beta^{-1} \ln \left(n / n_{i}\right)+\phi-\Delta \epsilon_{g}$, where $n_{i}$ is the material intrinsic density and $\Delta \epsilon_{g}$ is the bandgap narrowing of the considered band due to the doping. Eq. (1) is a fixed point equation for $h: h=F(h)$, where $F(h)$ is the rhs of (1). Because $h$ is a fixed point, $F^{N}(h)=h$ for all positive integers N. $F^{N}(h)$ contains $\left(3^{N+1}-1\right) / 2$ terms of which $3^{N}$ still depend on $h$. These $h$-dependent terms are all proportional to $W_{t o t}^{-N-1}$, where $W_{t o t}$ is the total scattering rate. For not too large $\mathrm{DF}$, the $h$-independent part of $F^{2}(h)$ is already a very good approximation of $h$ :

$$
\begin{aligned}
& h=-\beta \sum_{i} \partial_{r_{i}} \psi S_{v_{i}}^{-1} \\
& \quad+\beta \sum_{i, j}\left(\beta \partial_{r_{i}} \psi \partial_{r_{j}} \psi-\partial_{r_{i}} \partial_{r_{j}} \psi\right) S_{v_{i} S_{v_{j}}^{-1}}^{-1} \\
& \quad-\beta \frac{q}{\hbar} \sum_{i, j} \partial_{r_{i}} \phi \partial_{r_{j}} \psi S_{\partial_{k_{i}} S_{v_{j}}^{-1}}^{-1}+O\left(W_{t o t}^{-3}\right) .
\end{aligned}
$$

Eq. (2) shows that the preponderate DF are the gradient of the quasi-Fermi potential, the electric field, and surprisingly the second derivative of the quasi-Fermi potential. In Section III it is shown that in small $N I N$ structures the second derivative of the quasi-Fermi potential dominates, with the unfortunate consequence, that in such structures no natural TCAD mobility (in the sense of [1]) can be defined anymore.

As long as Eq. (2) is valid it can advantageously be used instead of the MC method to locally solve the BTE.

\section{B. Coupling}

Eq. (2) contains two unknowns: the electrostatic potential $\phi$ and the quasi-Fermi potential $\psi$. These two potentials can be 
determined by self-consistently solving the Poisson equation (PE) and the current-continuity equation (CCE). Therefore, the coupling with the full Boltzmann solver (Monte Carlo or direct solver) needs three steps. First, starting from a provisory solution of the PE and the CCE (e.g. drift-diffusion or energy-balance), the regions $\Omega_{s a}$, where Eq. (2) is valid, are determined. In the remaining regions $\Omega_{M C}$ the BTE is solved with the boundary conditions given by Eq. (2). Then, transport parameters (TP) (mobilities and diffusivities) are extracted in both regions $\Omega_{s a}$ and $\Omega_{M C}$ using the method described in [4]. Finally, the generalized drift-diffusion (GDD) equation [5] is solved everywhere using these new TP. The solution variables of the GDD equation are the electrostatic potential and the quasi-Fermi potential. These potentials are used again in the first step described above to determined the two regions $\Omega_{s a}$ and $\Omega_{M C}$. The three steps are iterated until convergence is achieved.

To determine where Eq. (2) is valid, a conservative criterion has been used: TP are not allow to change by more than $25 \%$ of their equilibrium value. Using the notation of [1], this criterion can be mathematically written as

$$
\begin{aligned}
& \mid \sum_{m, l=1}^{3}\left(\partial_{r_{m}} \phi \partial_{r_{l}} \psi M_{m, l, i, i}^{1}(r)\right)+ \\
& \sum_{m, l=1}^{3}\left(\left(\partial_{r_{m}} \psi \partial_{r_{l}} \psi-\beta^{-1} \partial_{r_{m}} \partial_{r_{l}} \psi\right) M_{m, l, i, i}^{2}(r)\right) \mid<0.25 \cdot D_{i i}^{e q},
\end{aligned}
$$

for the diffusivity and

$$
\begin{aligned}
& \mid \sum_{m, l=1}^{3}\left(\partial_{r_{m}} \phi \partial_{r_{l}} \psi M_{m, l, i, i}^{3}(r)\right)+ \\
& \sum_{m, l=1}^{3}\left(\left(\partial_{r_{m}} \psi \partial_{r_{l}} \psi-\beta^{-1} \partial_{r_{m}} \partial_{r_{l}} \psi\right) M_{m, l, i, i}^{4}(r)\right) \mid<0.25 \cdot \mu_{i i}^{e q},
\end{aligned}
$$

for the mobility. These criteria ensure the convergence of $F^{N}(h)$ for $N \rightarrow \infty$.

\section{RESULTS}

\section{A. NIN structure}

Eq. (2) has been used together with the current based oneparticle MC (CBOPMC) method [5] to compute transport parameters for the generalized Drift-Diffusion equation (GDD) [5]. Figs. 1, 2, and 3 show the bulk mobility compared to the mobility computed using Eq. (2). Fig. 7 illustrates the simple $N I N$ structure simulated in this paper. Fig. 8 shows the regions of the NIN structure where the semianalytical solution (Eq. (2)) is valid $\left(\Omega_{s a}\right)$ and those regions $\left(\Omega_{M C}\right)$ where the MC method must be used $(0.01 \mathrm{~V}$ bias). The criterion for the use of the semi-analytical solution has been described above. At the boundary between semi-analytical region and $\mathrm{MC}$ region the particles are consistently injected using Eq. (2). Fig. 5 shows the mobility in transport direction computed by the MC method. The shape of the mobility is dominated by the last term of Eq. (2), e.g. the electric field times the gradient of the quasi-Fermi potential as can be seen in Fig. $4\left(M^{1}, M^{2}, M^{3}\right.$, and $M^{4}$ are defined in [1]). Fig. 6 shows the diffusivity in transport direction computed by the MC method. Here, the shape of the diffusivity is astonishingly dominated by the second derivative of the quasiFermi potential (s. Fig. 4), which has, to our best knowledge, never been mentioned as a variable to parameterize models for the mobility and/or the diffusivity.

The main advantage of this new coupling scheme is clear. Only the $N I$ junctions must be simulated using MC, whereas a semi-analytical solution exists in the highly doped regions and in the center of the device. The simulation time saved here is of about a factor of ten. The drawback is that the ISO contained in Eq. (2) must be recomputed after each iteration. The cost for these computations is, however, of only $1 \%$ of the total computation time.

\section{B. Discretization-related problems}

In this work, the discretization of the band structure for the semi-analytical solution was the same as the one used in the MC simulator. The first Brillouin zone (BZ) is discretized in small cubes with edges of the size $1 / 96$ of the BZ. The velocity and the mass (first and second derivative of the band structure) are assumed constant in each box, whereas the energy is linearly interpolated. This kind of discretization is usual in MC simulators, but has a main drawback when directly computing transport parameters. At thermodynamic equilibrium (TDE) the Einstein relation is not exactly fulfilled. There is usually a discrepancy of about $3 \%$ to $5 \%$. Furthermore, an exact relation between $M^{1}$ and $M^{4}$ is also not perfectly fulfilled. The consequences of these numerical errors will become clear below.

\section{Surface scattering problem}

Surface scattering was taken into account using the model proposed in [6], i.e. allowing reflexive and specular scattering at the interfaces. These special kinds of scattering are responsible for the violation of the Einstein relation at TDE, and should be avoided when using the new coupling scheme (e.g. by using a scheme based on elastic scatterings proportional to the component of the electric field normal to the interface).

\section{Space-charge regions and violated Einstein relation}

In the two previous subsections, different causes of the violation of the Einstein relation were discussed. These violations have a visible impact on the course of the quasiFermi potential, but astonishingly not on the terminal current. This problem is not directly related to the hybrid method, but it was discovered during its validation. In the space-charge regions (i.e. the $N I$ regions), the electric field can be very high even at TDE. From the CCE, the quasi-Fermi potential 




Fig. 1. Bulk transport parameters: comparison between MC and Eq. (2) (without doping).

can be rewritten as

$$
\psi(x)=\int_{0}^{x}\left(1-\beta \frac{D_{x x}\left(x^{\prime}\right)}{\mu_{x x}\left(x^{\prime}\right)}\right) E_{x}\left(x^{\prime}\right)+v_{x}\left(x^{\prime}\right) d x^{\prime}+C .
$$

At TDE, the second term inside the integral vanishes (i.e. the velocity is zero everywhere). If the Einstein relation is fulfilled, the term in parenthesis also vanishes leading to a constant quasi-Fermi potential. If the Einstein relation is violated, the effect will be the bigger the larger the intensity of the electric field is. Therefore, in the space-charge region, even a small violation of the Einstein relation will have a visible repercussion on the quasi-Fermi potential. This issue can only be solved using a better discretization of the BZ and a longer MC simulation time.

\section{E. Decreased efficiency of statistical enhancement}

When simulating NIN structures as shown in Fig. 7 using no hybrid method but only MC, one usually uses some kind of statistical enhancement to have the same statistics in the intrinsic region as in the highly doped regions. With the hybrid method, statistical enhancement can only be used in the MC region $\left(\Omega_{M C}\right)$, i.e. in the region where the electric field is quite high and where only few scattering events occur. Because of these few scatterings, the effect of statistical enhancement is highly reduced, because the path of the generated statistical particles is increasingly correlated. Thus, one should very carefully assess the effect of statistical enhancement when using it together with the hybrid method. It could generate a non-negligible computational overhead.

\section{CONCLUSION}

A semi-analytical method has been developed to solve the BTE in regions where the MC method is particularly inefficient. This method can be easily coupled with the MC technique (or a direct BTE solver) using the CBOPMC method. It allows to understand under which well-defined conditions the popular transport models (Drift-Diffusion and Hydrodynamic) are still valid, and allows to select the proper DF to parametrise models for the mobility and the diffusivity. Still open problems

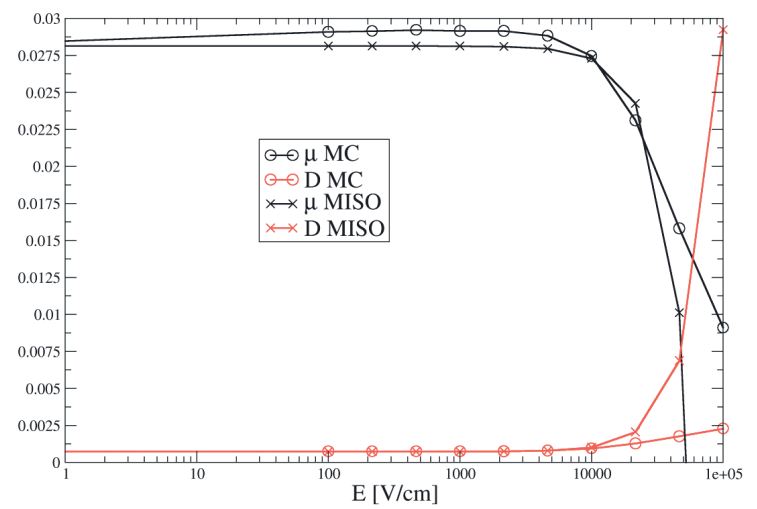

Fig. 2. Bulk transport parameters: comparison between MC and Eq. (2) using a doping concentration of $10^{18} \mathrm{~cm}^{-3}$.

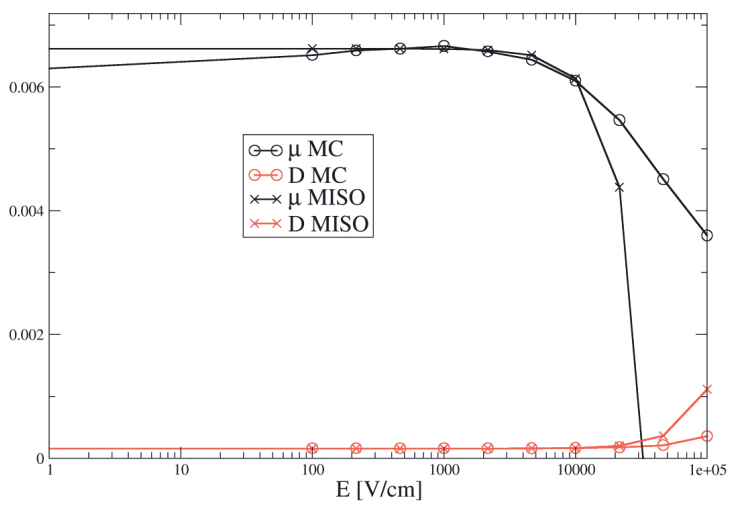

Fig. 3. Bulk transport parameters: comparison between MC and Eq. (2) using a doping concentration of $10^{20} \mathrm{~cm}^{-3}$.



Fig. 4. Contribution of the three DF of Eq. (2) to the mobility and the diffusivity in the NIN structure as function of position $x$. 


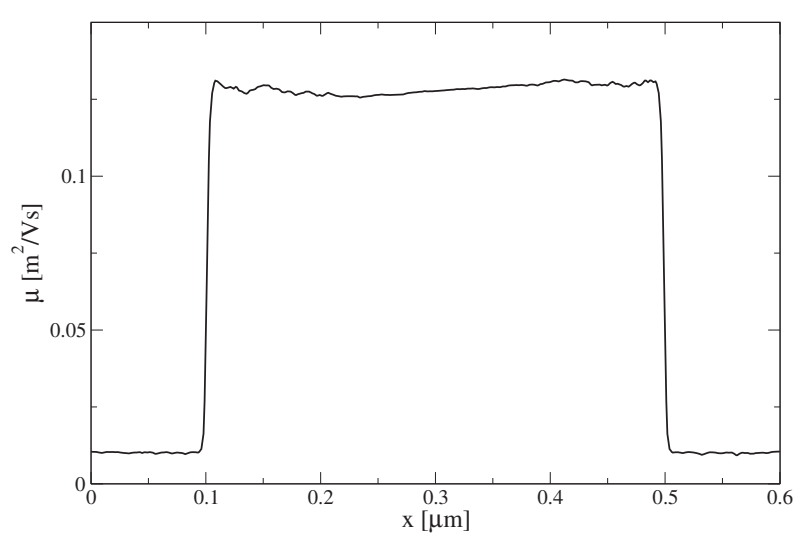

Fig. 5. Mobility extracted from a MC simulation in the $N I N$ structure as function of position $x$.

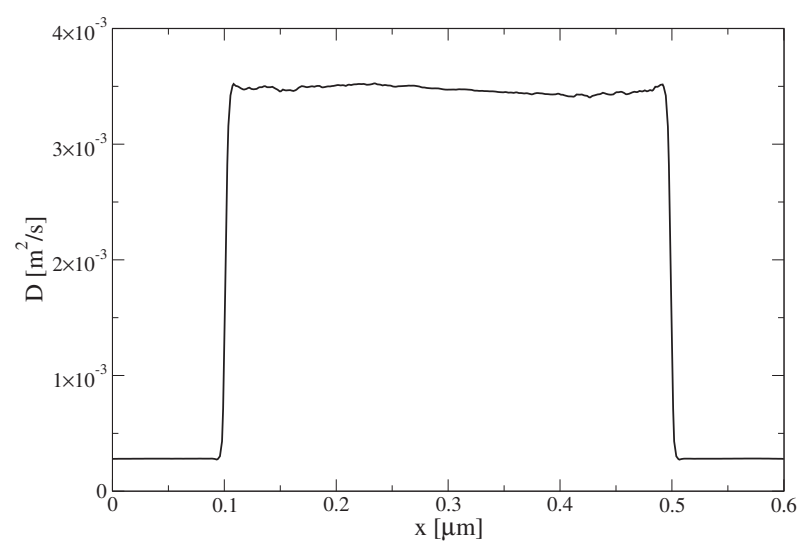

Fig. 6. Diffusivity extracted from a MC simulation in the $N I N$ structure as function of position $x$.

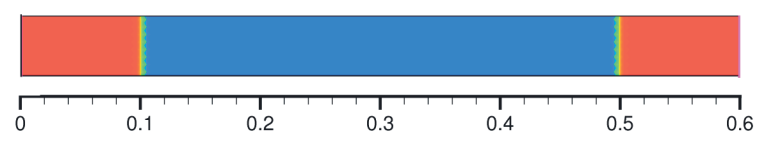

DopingConcentration(MCDopingConcentration) $\left[\mathrm{cm}^{\wedge}-3\right]$
$1.0 \mathrm{E}+19$
$1.8 \mathrm{E}+18$
$3.3 \mathrm{E}+17$
$6.0 \mathrm{E}+16$
$1.1 \mathrm{E}+16$
$2.0 \mathrm{E}+15$

Fig. 7. Geometry and Doping profile of the simulated $N I N$ structure.

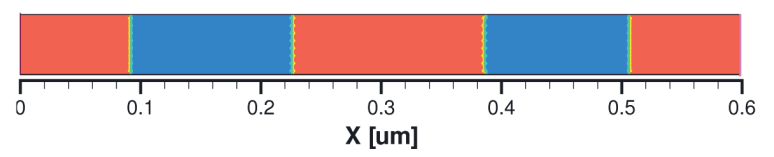

Fig. 8. Semi-analytical regions (red) and MC regions (blue) automatically detected using the criterion eqs. (3)-(4). are mainly related to the poor discretization of the band structure. A better discretization should be found.

\section{ACKNOWLEDGEMENT}

The authors are grateful for the financial support by the Swiss National Foundation (SNF 200021-109393 NEQUATTRO-2) and by the EU project IST-4-026828-IPPULLNANO.

\section{REFERENCES}

[1] S. C. Brugger, V. Peikert, and A. Schenk, "Exact method to solve the Boltzmann equation to any order in the driving forces: Application to transport parameters," Physica Status Solidi (c), vol. 5, no. 1, pp. 86-89, 2008 .

[2] S. C. Brugger, A. Schenk, and W. Fichtner, "Moments of the inverse scattering operator of the Boltzmann equation: Theory and applications," SIAM Journal on Applied Mathematics, vol. 66, no. 4, pp. 1209-1226, 2006. [Online]. Available: http://link.aip.org/link/?SMM/66/1209/1

[3] S. C. Brugger, "Computation of semiconductor properties using moments of the inverse scattering operator of the Boltzmann equation," Ph.D. dissertation, ETH Zürich, published at Hartung Gorre Verlag, Konstanz, 2005. [Online]. Available: http://ecollection.ethbib.ethz.ch/show?type=diss \&nr $=16376$

[4] S. C. Brugger and A. Schenk, "Universal method for extracting transport parameters from monte carlo device simulation," IEEE Trans. Electron Devices, vol. 54, no. 11, pp. 3092-3096, 2007.

[5] — , "New one-particle Monte Carlo method for nanoscale device simulation," in Technical Proceedings of the 2006 NSTI Nanotechnology Conference and Trade Show, vol. 1, Boston, MA, USA, 2006, pp. 673676.

[6] E. Sangiorgi and M. R. Pinto, "A semi-empirical model of surface scattering for Monte Carlo simulation of silicon n-MOSFET's," IEEE Transactions on Electron Devices, vol. 39, pp. 356-361, 1992. 